\title{
Research on the Intelligent Service System of University Mobile Digital Library
}

\author{
Jia-Xin Yue ${ }^{1}$, Ai-Min $\mathrm{Pu}^{1}$, Xu Huang ${ }^{2}$ \\ ${ }^{1}$ Sichuan Film and Television University, Chengdu 610000, Sichuan,China; \\ ${ }^{2}$ Teachers College of Chengdu University, Chengdu 610000, Sichuan, China
}

Keywords: colleges and universities; mobile digital library; wisdom service

\begin{abstract}
The mobile digital library is the result of the informatization construction of the university library. The intelligence service system is the reader service form of the mobile digital library. Starting from this, the article first analyzes the connotation of smart services, and then introduces the current status of mobile digital libraries in colleges and universities, including the total number of digital resources, the low level of reader service intelligence, followed by user centers, value utilization, and convenience. From the perspective, we discussed the main points of building a smart service system. Finally, we put forward suggestions on how to build a smart service system, including technological innovation as a prerequisite, resource enrichment as a key point, personalized service as a goal, and collaborative service as a development.

As the cultural hub and information center of the university, the library also undertakes the dual tasks of education and scientific research and plays a fundamental role in the students' learning, growth, and development. With the continuous development of information technology, university libraries have gradually developed from offline to online. Most colleges and universities have built mobile digital libraries, greatly expanding the scope of the library's functions. Reader service is the most basic service for university libraries. The arrival of the information age and the construction of mobile digital library put forward higher requirements for the readers' service capabilities of university libraries. Traditional reader service has become increasingly difficult to meet the needs of teachers and students, and smart services have become a reliable choice. Based on this, the article made a corresponding study on the construction of the intelligent service system of college mobile digital library.
\end{abstract}

\section{Intelligence service analysis}

The traditional university library service about reader is offline service, and the artificial service is the main type. Due to the double restriction of site and time, the service efficiency is low, which seriously affects the actual role of the library in education and scientific research. The wisdom service and the mobile digital library are accompanied by a new reader service form. It takes the mobile receiving terminal as the service carrier and the digital information technology as the service medium, which can well meet the different types of service needs of different readers. Compared with human services, smart services have the following characteristics: First, the open features, the traditional services are generally closed, at a specific time, a specific space for specific personnel to complete, and smart services are Break through the limitations of time and space, teachers and students can accept readers' services at any time and any place under the premise of having a network; secondly, intelligence characteristics. Traditional services are mostly mechanical, readers put forward corresponding service demands, librarians provide corresponding reader services, services are lack of initiative, lack of targeted, low service value density, intelligence services rely on information technology, can analyze The preference of readers gives them active and personalized reader services, and the service intelligence is greatly improved. 


\section{Development Status of College Mobile Digital Library}

\subsection{The total number of digital resources is too low}

It is undeniable that the current construction of mobile digital libraries has been carried out in various universities and has achieved very good results, but at the same time it is necessary to note that there are still many problems in the construction of mobile digital libraries in colleges and universities, the most typical It is the low total amount of digital resources. The digital resources of books are the traditional form of paper resources. The common formats are txt, doc, pdf, etc. Digital resources are the premise of teacher-students' electronic reading. The quantity and type of digital books resources are directly related to the development status of college mobile digital libraries. The current college digital library construction still focuses on the informationization of the management system. Teachers and students rely on the digital platform to find basic information about books, such as location, quantity, loan history, and reader evaluation, but most college books The library did not effectively integrate library resources in the museum, which made it difficult for students to quickly and easily download resources from the mobile digital library, which seriously affected the effectiveness of mobile digital library construction.

\subsection{Low level of service intelligence for readers}

Regardless of the form of library, reader service is its most basic work content. The quality of reader services is related to the final results of the construction of mobile digital libraries, and highlighting reader services is also a manifestation of the people-oriented basic requirements for mobile digital library construction. As far as the current status of mobile digital library construction is concerned, although reader services have achieved informatization, there is still a long way to go from the perspective of intelligentization. Intelligent services require mobile digital libraries to analyze readers' reading preferences based on readers' borrowing records, online messages, and community interactions, thereby providing readers with intelligent services. This has high requirements for mobile digital libraries. It is a technical level that requires the mobile digital library to be able to apply the big data technology. However, the total number of libraries currently using big data technology in mobile digital libraries is not large, which affects the actual effect of readers' intelligent services.

\section{The key points of building a smart service system for college mobile digital library}

\subsection{User-centric}

The construction of mobile digital libraries in colleges and universities will eventually serve the majority of readers inside and outside the school, especially the teachers and students in the school. Therefore, the basic principle to be followed in the construction of a smart service system is user-centered. First of all, in terms of the concept of building smart services, college mobile digital libraries need to follow the reader center concept and proceed from the actual needs of readers to carry out specific construction work, including optimization of management systems, enrichment of library resources, and improvement of service processes. Secondly, as far as the optimization of smart services is concerned, college mobile digital libraries need to pay attention to the user experience, and regularly collect educators' and students' advice on smart services through electronic questionnaires, and make necessary adjustments and optimizations according to the recommendations.

\subsection{Use is key}

Book resources are different from general resources, and they have unlimited use. With the increase in the number of users and frequency of use, the social value of library resources is also increasing geometrically. Therefore, in the construction of mobile digital library reader services in colleges and universities, it is necessary to use them as the key. Strengthening the utilization efficiency of library resources is also the key point of the construction of a smart service system. Specifically, colleges and universities can build corresponding university library alliances in the construction of mobile digital library and communicate with each other through the form of cooperation. This can not only effectively enrich student's reading choices, but also significantly 
increase the number of students. The utilization efficiency of library resources for books, the strengthening of the reuse of resources and the reduction of waste of resources are also the objective needs of the era of low-carbon economy.

\subsection{Take convenience as the goal}

The practice of digital platform construction shows that complicated interface settings and cumbersome operation processes have a great negative impact on user viscosity. With increasing emphasis on efficiency today, users are very much looking for the most desired information in the shortest possible time. Therefore, the mobile digital library must pay attention to the convenience of the service flow in the construction of the intelligent service system, and reduce readers' access to intelligent services. Cost, improve the efficiency of smart services. Therefore, the principle of convenience is also one of the key points in the construction of a smart service system for mobile digital libraries in colleges and universities. In the interface setting or the operating behavior of the application terminal, it should be as simple and optimized as possible so that the user can play the value of the digital library well without having to master the complicated techniques. Not only that, in the construction of a smart service system, it is necessary to take into account the compatibility of the user terminal and to reduce the amount of memory that it occupies for the user terminal as much as possible.

\section{Strategies for the Construction of a Smart Service System for College Mobile Digital Libraries}

\subsection{Prerequisite for technological innovation}

The birth and development of information technology is the prerequisite for the establishment and development of college mobile digital library. Similarly, the further development of information technology is also a necessary guarantee for the mobile digital library to continuously serve teachers and students. The wisdom service is different from the general reader service. It is characterized by intelligence, comprehensiveness, and professionalism, and has a very outstanding service value. In the construction of a smart service system, it is necessary to take technical innovation as a precondition, especially the Internet of things technology and big data technology. First of all, as far as the technology of Internet of things is concerned, it is a technology for exchanging information between the external world and the network world. It has outstanding value in the construction of a smart service system, such as connecting student's library card with a network system through radio frequency identification. Get student's borrowing information, etc. For another example, the mobile digital library can develop the mapping function. Readers only need to take out the cover of the book to carry out identification and search within the platform, which effectively reduces readers' book search time. Second, in terms of big data technology, unlike general data technologies, big data technology can not only deal with structured data, but also can handle semi-structured and unstructured data. The application in the mobile digital platform can well analyze the reading needs of readers. Big data has such characteristics as huge total quantity and many types. Distributed cloud computing is the basic path for improving the processing speed and processing efficiency of big data. Because of the unique value of big data technology, technological innovation is the precondition for the construction of a smart service system.

\subsection{Focus on resource enrichment}

In the construction of a smart service system for college mobile digital libraries, the type and quantity of library resources are important factors affecting the construction of smart services. At present, mobile digital libraries in colleges and universities generally face the problem of less e-book resources, which affects the actual effect of smart services. Therefore, colleges and universities in the construction of a smart service system focus on resourcefulness. First of all, colleges and universities should do a good job in the digitization of library resources in schools. Digitization methods include text entry, scanning, uploading, and other forms. Teachers should use suitable methods according to the types of library resources. For example, text-only books can be used for text entry. The way for students to read, edit, and for books with more charts, you can use scanning; second, colleges and universities can build libraries with other schools in the form of 
alliances, to expand the channels for students to borrow books, it lays a solid foundation for students to use mobile digital library to acquire more book resources.

\subsection{Targeting Personal Service}

In traditional library services, the library provides undifferentiated services to all readers. With the continuous development of information technology and the continuous improvement of the level of reader service requirements, it has become increasingly difficult for non-differentiated reader services to meet the actual needs of readers. Personalized services have become the main trend of library reader service development. For the university library, the personalized service in the construction of the intelligent service system can proceed from the following points: First, it highlights the specialization of library resources. Colleges and universities, especially comprehensive universities, have many internal specialties. Students in different majors have different preferences and needs in book borrowing. Libraries need to classify digital book resources according to their specialization to facilitate the search of students from different disciplines. Second, strengthen the application of big data technology. Different students have different reading preferences, and the mobile digital library's account password login mechanism enables the platform to use big data analysis techniques to analyze students' browsing trajectories to derive their reading preferences, such as preference for history - modern history - - Character biographies, etc., to provide readers with relevant book information in a timely manner, which can improve students' satisfaction. In the end, the mobile digital library platform needs to provide students with the authority to build a personal library, and students can use their own actual conditions. The situation is to manage the information of library resources, and at the same time, according to their own needs, a book information warehouse is established to meet the actual needs of reading activities.

\subsection{Development with collaborative services}

As an integral part of colleges and universities, library resources have a lot to do with the professional attributes of universities. For example, colleges and universities in the humanities and social sciences of normal colleges have more books, while universities in science and engineering have more books in natural sciences and engineering equipment. This kind of distribution of book resources makes it difficult for a single university library to effectively meet the increasing information needs of students, and offline collaboration is limited by time and space. For this reason, mobile digital libraries have a very prominent application value. Colleges and universities need to build a library alliance, especially college gathering areas, such as Nanjing Xianlin University City and Wuhan Optics Valley University City, to meet the reading needs of college students in the region with the sharing of library resource information, and effectively improve the service quality. The collaborative service in the smart service system has dual connotations. It refers not only to the collaborative services between university libraries but also to the collaborative services between the university library and the government. At present, many of the policies promulgated by the State are closely related to the employment, examination and examination of college students. Libraries need to strengthen the integration of relevant information and provide students with all necessary links to government information so that students can acquire relevant future developments. The various information resources, while the university library also need to provide students with employment and other social and public affairs to provide a corresponding information platform, reduce the time cost of students in the transaction processing, improve the actual service efficiency of mobile digital library intelligence service system.

\section{Conclusion}

With the continuous development of information technology and the increasingly close integration with human life, digital library construction has become an inevitable requirement for its development. What is equally important to the construction of digital libraries is the change in reader service models, specifically the transformation from traditional manual services to intelligent services in the information age. Intelligent services are mainly characterized by intelligence. Not only can services be provided to readers more quickly, but also the services are more targeted, and the satisfaction of teachers and students' reading needs is of considerable value. Therefore, colleges 
and universities in the construction of mobile digital library should highlight the construction of a smart service system.

\section{References}

[1] Guo Rongmei. Wisdom Library: Effective Support for Campus Cultural Brand Cultivation [J]. Lantai World, 2013 (35): 136-137

[2] Su Shuangsheng. Study on the Service Model of Higher Vocational College Library Facing the Construction of Harmonious Campus Culture[J].Henan Journal of Library Science, 2010, 30 (3): 77-78

[3] Zhang Yan. Constructing a New Model of Smart Library Services to Serve the Construction of Campus Culture [J]. Chinese Information, 2015 (5): 44-46 